\title{
Paroxysmal atrial flutter and fibrillation Induction by carotid sinus compression and prevention by atropine
}

\author{
Nabil El-Sherif \\ From the Cardiology Department, Faculty of Medicine, Cairo University, Cairo, Egypt, U.A.R.
}

Two patients with paroxysmal atrial flutter and fibrillation are reported. Episodes of the arrhythmia were repeatedly induced by carotid sinus compression and always started by an early coupled atrial beat. The findings at the onset, course, and termination of the episodes could be interpreted in favour of either the focal theory or re-entry mechanism. Analysis of the recovery phase of episodes of atrial fibrillation revealed evidence in favour of re-entrant activity. On the other hand, the occasional demonstration during episodes of atrial fibrillation of two different types of regular tachysystole with opposite propagation patterns may be explained by the concomittant operation of two ectopic foci. The report suggests that vagal-induced bradycardia may underlie the episodes of arrhythmia in some patients with paroxysmal atrial flutter and fibrillation. Prevention of excessive vagal reflexes by atropine medication offers a physiological approach to the management of these cases.

The role of vagal stimulation in precipitating atrial flutter and fibrillation in experimental animals is well known. Clinical observations showing similar phenomena during carotid sinus compression or other vagal reflex mechanisms are, however, very rare (Scherf, Cohen, and Rafailzadeh, 1966). We have recently observed two cases of paroxysmal atrial flutter and fibrillation where the episodes could be repeatedly elicited by carotid sinus compression while they were successfully prevented by atropine. Critical analysis of the onset, course, and termination of the episodes was carried out. Study of paroxysmal type of atrial flutter and fibrillation usually offers an insight into the mechanism of the arrhythmia which may have some bearing on the nature of the disorder in general. Though the reported observations could be interpreted in favour of either the focal theory or re-entry mechanism, the clinical setting at the onset of the disorder offers a physiological approach to the management of some patients with paroxysmal atrial flutter and fibrillation, no matter what mechanism proves to be operating.

\section{Case reports}

Case I A 52-year-old man with emphysema, cor pulmonale, and early right-sided failure was

Received 7 January 1972. first seen in March 1970. The patient gave a history of recurrent episodes of rapid palpitation in the last 6 months. The conventional electrogram revealed atrial fibrillation, low voltage of the extremity leads, and moderate clockwise rotation in the horizontal plane. The patient was treated for chest infection and kept on antifailure measures. He was seen 6 months later when he was no longer on antifailure regimen for the last three months. Examination revealed regular sinus rhythm with no evidence of congestive failure. Frequent episodes of rapid palpitation had recurred since the last examination. The patient was thought to have paroxysmal atrial fibrillation and the trial to induce the paroxysms by carotid sinus compression was successful. A total of 28 episodes could be induced in two sittings. The episodes lasted from one second (Fig. ID) up to several hours and always started with an early coupled atrial beat with a fixed coupling interval of $0.36 \mathrm{sec}$. Not all atrial ectopic beats were followed by the onset of fibrillation. Aborted episodes consisting of two ectopic discharges in rapid succession interpolated between two sinus impulses were occasionally seen (Fig. IC and E). Frequently carotid sinus compression gave rise to sinus bradycardia that lasted for many cycles before the onset of the episode (Fig. IB). Uncommonly, the episode rapidly followed carotid sinus compression after a few sinus intervals, the last of which was excessively prolonged (Fig. IA). In a few instances carotid sinus compression gave rise to a series of fixedly coupled atrial beats making a short run of bigeminy, the last of which started the episode (Fig. IC). 
During the episodes, the atrial wave form as recorded by the oesophageal VE lead was completely disorganized as is typical for atrial fibrillation (Fig. 2D). Not uncommonly, however, a clearly defined atrial wave form of regular tachysystole was seen, particularly before the termination of the episode. Two types of atrial tachysystole could be identified. The first type (A) inscribed positive deflections in lead II and an rS configuration in the VE lead, suggesting a focus near the sinoatrial (SA) node (second half of record B in Fig. 2). Type (A) tachysystole showed a frequency of 280-300 a minute and occurred more frequently than the other type (B) which showed practically the same rate but inscribed negative deflections in lead II and an $\mathbf{R}$ configuration in the VE lead which would denote a focus of origin near the atrioventricular (AV) node (Fig. 2A). Occasional shifts between the two types were observed with many intermediate wave forms (Fig. 2C).

The termination of episodes of atrial fibrillation was critically analysed. Twenty-four instances were available for study and the following patterns were observed.

a) In 3 instances, atrial tachysystole of type (A) ended abruptly without lengthening of the last ectopic interval (Fig. 3A).

b) In 4 instances there was definite lengthening of the last ectopic interval after a period of type (A) tachysystole (Fig. 3B).

C) In II instances type (A) tachysystole ended with a type (B) ectopic impulse after a relatively prolonged interval (Fig. IC).

$\Delta$

FI . I Case I. Paroxysmal atrial fibrillation. Several episodes were induced by carotid sinus compression (C.S.C.). Note brief episodes in records $(C)$ and $(D)$. The episodes always started by an early coupled atrial beat.

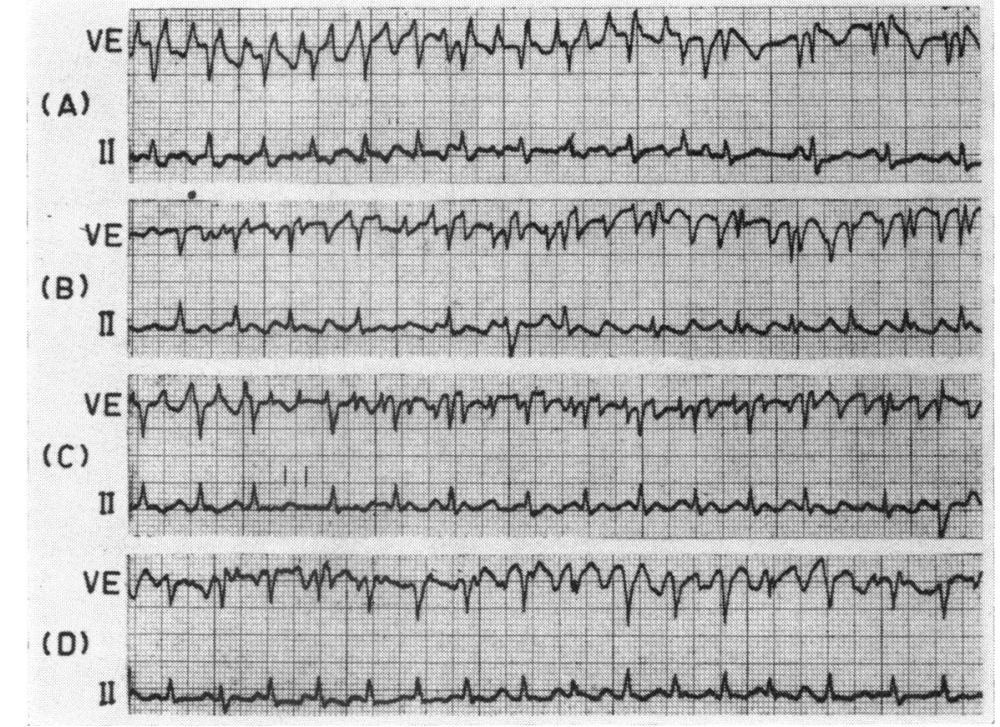

FIG. 2 Case I. Record $(A)$ and the second half of record $(B)$ show two types of regular tachysystole inscribing negative and positive deflections in lead II, respectively. Record $(C)$ illustrates shift between the two types with multiple intermediate wave forms. Record $(D)$ reveals disorganized atrial wave form of classic fibrillation.

d) In 3 instances the above arrangement occurred without undue lengthening of the last ectopic interval (Fig. $3 \mathrm{E}$ and $\mathrm{F}$ ).

e) In 2 instances type (B) tachysystole ended by a type (A) ectopic impulse after a relatively prolonged interval (Fig. 2A).

f) In I instance there was alternation between ectopic beats of types (A) and (B) (Fig. 3D).

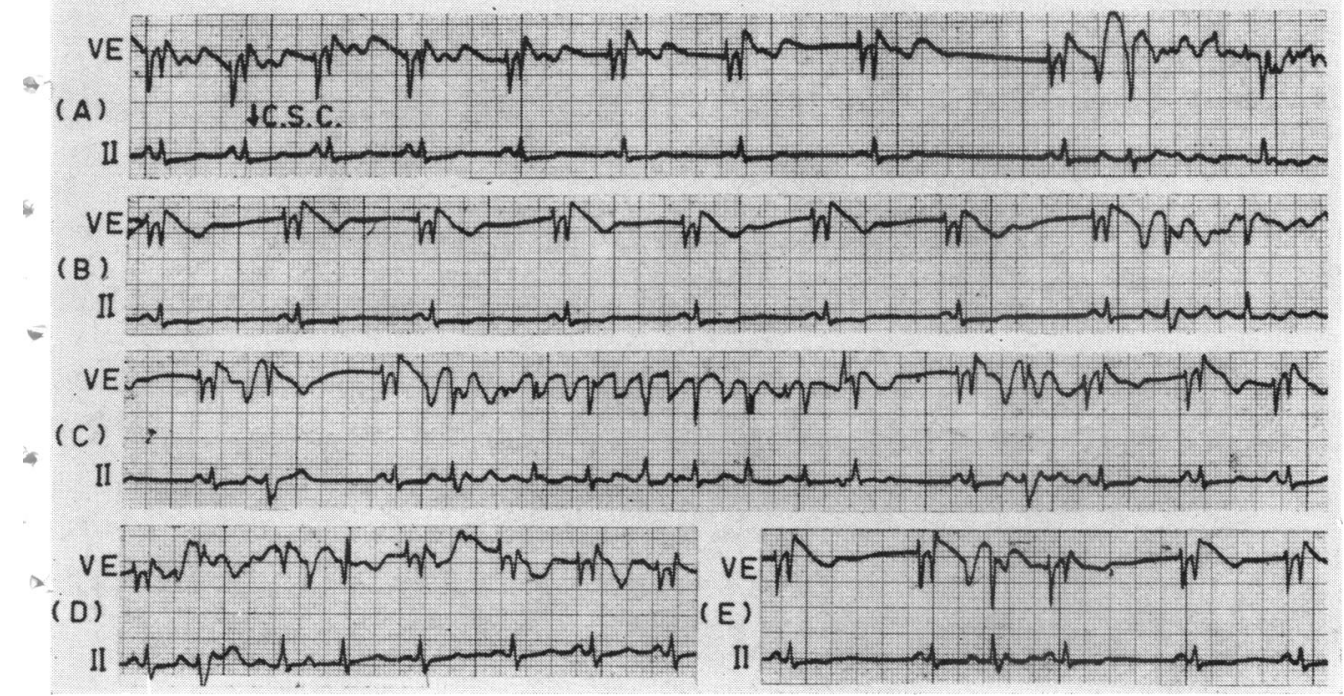


(A)
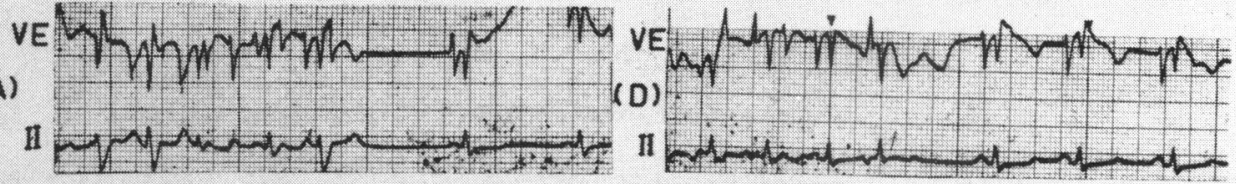

(B)
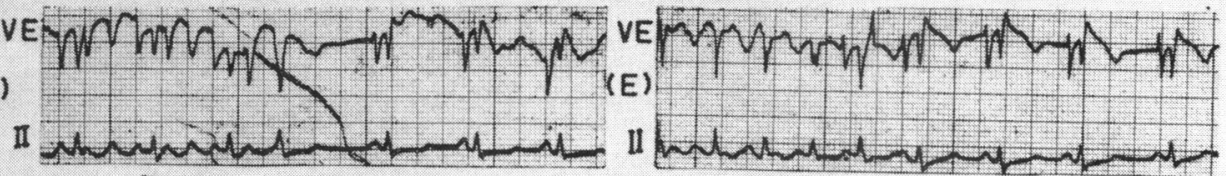

(C)
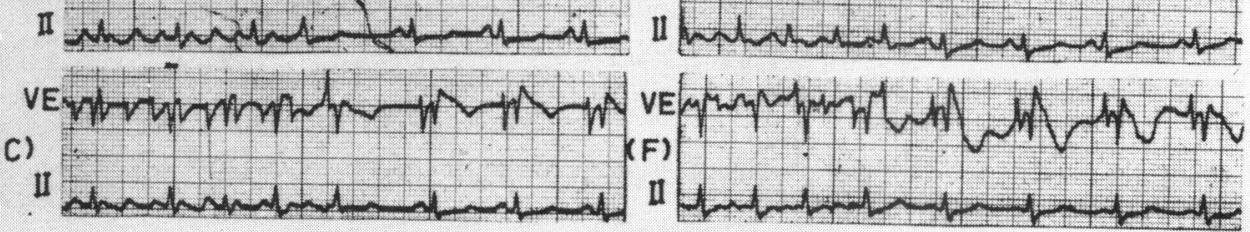

FIG. 3 Case I. Different patterns of termination of episodes of atrial fibrillation. See text for explanation.

In 20 out of 24 instances of recovery phases analysed, the postectopic pause exceeded the next sinus cycle length. In 4 instances, however, this was significantly shorter (Fig. $3 \mathrm{E}$ and $\mathrm{F}$ ).

Atropine sulphate in a dose of $\mathrm{I} \mathrm{mg}$ was administered intravenously during cardiographic monitoring. The drug resulted in moderate sinus tachycardia and the episodes were no longer elicited by carotid sinus compression. Oral atropine medication was started with $\mathrm{I} \mathrm{mg}$ every 6 hours, which was later increased to I mg every 4 hours, with no other medication. The patient was followed for the next 6 months and the episodes of palpitation did not recur.

Case 2 A 58-year-old man was first seen in September 1970 with an attack of rapid heart action associated with conspicuous dyspnoea and severe anginal pains. The patient gave a history of similar attacks of palpitation of one year's duration that lasted from a few minutes up to several days. The conventional electrogram showed regular supraventricular tachycardia with a rate of $I 50$ a minute and moderate ST-T changes in inferolateral surface leads. Carotid sinus com-

FIG. 4 Case 2. A short episode of atrial flutter induced by carotid sinus compression (C.S.C.). The episode starts by an early coupled atrial beat (marked $X$ ). pression applied in a trial to stop the attack resulted in irregular AV block, revealing that we were actually dealing with atrial flutter with $2: 1$ AV response. The patient was seen one day later for a trial to revert the arrhythmia, but he was found in sinus rhythm. The patient was diagnosed as paroxysmal atrial flutter and under cardiographic monitoring a trial was made to induce the arrhythmia by carotid sinus compression. Several episodes that lasted from a few seconds ( 12 seconds in Fig. 4) up to several hours could be elicited in more than one sitting. The episodes always started with an early coupled atrial beat (marked X in Fig. 4 and 5). The episodes followed carotid sinus compression after a brief (Fig. 4) or long interval of sinus bradycardia (Fig. 5C); or after a period of bigeminy caused by regular occurrence of fixedly coupled atrial beats, the last of which started the paroxysm (Fig. 5B). Not infrequently the episode started with a short period of disorganized atrial wave form as shown in the VE lead before the development of a clearly defined wave form of atrial flutter (Fig. $5 \mathrm{C}$ ). Records (D) to (F) in Fig. 5 were obtained during one of the prolonged episodes. Record (D) shows positive flutter waves with a rate of 300 a minute. Record (E) illustrates transition from positive flutter waves in the first half of the record to more rapid negative flutter waves in the second half; the latter is clearly shown in record $(F)$, with a rate of 360 a minute.

Intravenous administration of I $\mathrm{mg}$ atropine sulphate resulted in moderate sinus tachycardia and the episodes were no longer elicited by carotid sinus compression. The patient was kept on oral

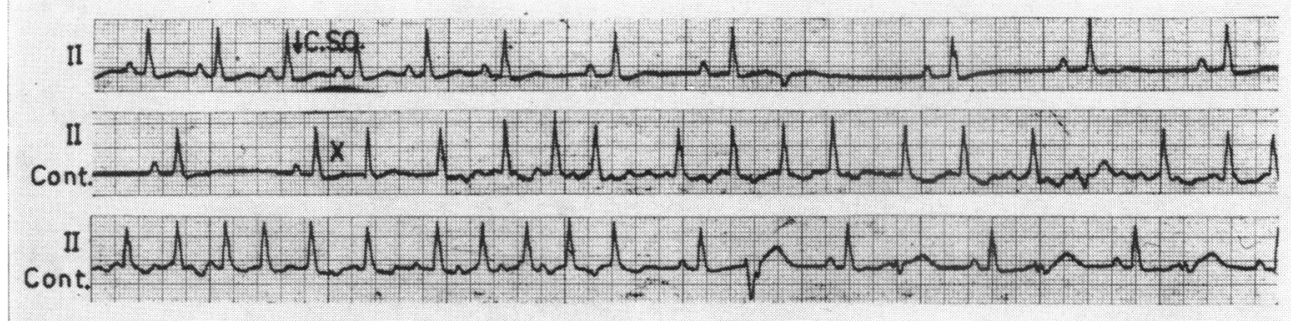


FIG. 5 Case 2. Records $(A)$ to $(C)$ illustrate three trials to induce atrial futter by carotid sinus compression (C.S.C.). The trial failed in record $(A)$ and succeeded in $(B)$ and $(C)$. Note that the episodes always started by an early coupled atrial beat (marked X). Record (D) shows positive flutter waves that gradually shift in record $(E)$ to a more rapid negative flutter waves well illustrated in record $(F)$.

atropine and was regularly observed for the next four months. He was asymptomatic and the episodes were not reported.

\section{- Discussion}

Re-entry and focal activity are the basic concepts of all theories of atrial flutter and fibrillation (Hecht et al., 1953). From the theoretical point of view, both mechanisms can produce the arrhythmia; the controversy usually arises from the interpretation of available clinical and experimental evidence.

To the proponents of re-entry the repeated

- induction of atrial flutter and fibrillation by carotid sinus compression is explained by the effects of both increased vagal tone (Alessi et al., 1958) and the bradycardia per se (Han et al., 1966; Goel and Han, 1970) in increasing the degree of asynchrony of recovery of excitability in the atrium. This favours focal - re-excitation of already repolarized fibres by the flow of current between these and neighbouring fibres which are still depolarized, and results not only in closely coupled ectopic beats but also in repetitive activity including atrial flutter and fibrillation (Han et al., 1966; Goel and Han, 1970).

Analysis of the propagation pattern of the last few beats of fibrillation in Case I may reveal evidence in favour of re-entrant activity. In more than half of the recovery phases analysed, the propagation pattern of the last beat of an episode showed a diametrically opposite direction to the pattern of previous beats which have been similar to each other. This observation in addition to lengthening of the last ectopic interval can be explained by assuming that the penultimate ectopic impulse has slowly propagated through one of the nodal centres and re-entered the atria in a large circus movement. Fibrillation episodes showing type (A) tachysystole near the termination probably ended with re-entrant activity in the AV node, while episodes showing type (B) tachysystole ended with re-entry into the sinoatrial node. Similar propagation patterns during the recovery phase of electrically induced atrial fibrillation in dogs have been reported by Sano and Scher (1964) and were explained on the same lines.

To the proponents of the focal theory, the finding in the present report of consistent initiation of episodes of atrial flutter and fibrillation by closely coupled ectopic atrial beats is explained on the basis of stimulation of the atria during the vulnerable phase. It has been shown in clinical records that the coupling interval of atrial ectopic beats which initiated atrial fibrillation is significantly shorter than of those which did not, and that the episodes frequently followed fixedly coupled ectopic beats only after a relatively long preceding cycle length (Killip and Gault, 1965; Bennet and Pentecost, 1970); both findings may be taken as evidence that a vulnerable period exists in the human atria. There is some question, however, as to when an atrial vulnerable phase is reached, for it is usual that not all atrial musculature regain their full excitability at the same time (Hecht, 1970). Both increased vagal activity and bradycardia per se, by further increasing the range of dis- 
parity of the refractory periods in the atrium, will increase the chance for an early coupled atrial ectopic beat to fall in the vulnerable phase of some of the atrial musculature.

Though atrial fibrillation may arise as a result of discharge from a single focus, other foci may be recruited and extinction of all foci becomes necessary if the arrhythmia is to be terminated (Scherf, I 966). The demonstration of types (A) and (B) tachysystoles in Case I may be interpreted as a manifestation of two different ectopic foci firing concomitantly. The disorganized atrial wave form of classic fibrillation can be explained by (a) varying pattern of conduction from both foci, (b) varying pattern of atrial propagation of each of the rapid ectopic discharges, and (c) variable degree of atrial fusion complexes between discharges of both foci. On the other hand, the short periods of regular tachysystoles observed in the case can be explained by (a) 'manifest' activity in only one focus; the other being temporarily suppressed (either becoming subthreshold or only very limitedly propagated), (b) regular pattern of conduction from the focus, and (c) regular pattern of atrial propagation of repetitive ectopic discharges. The different terminations observed in the case can also be explained on the basis of operation of two different foci. On similar lines the observed shift between two different types of atrial flutter in Case 2 can be explained by the role of the primary focus of atrial flutter in inducing a more rapid discharge in another focus; the latter gradually overdrove and suppressed the former.

In contrast to the controversial theoretical interpretation, analysis of the clinical findings offered a clear approach to the management. Thus, whatever the electrophysiological mechanism by which vagal-induced bradycardia initiates episodes of arrhythmia in some patients with paroxysmal atrial flutter and fibrillation, the fact remains that prevention of excessive vagal reflexes forms the basis of a successful therapeutic regimen. In this connexion, atropine medication will be a more physiological approach compared to electroconversion and antiarrhythmic agents, which should be reserved for established forms of the disorder.

\section{References}

Alessi, R., Nusynowitz, M., Abildskov, J. A., and Moe, G. K. (1958). Non-uniform distribution of vagal effects on the atrial refractory period. American fournal of Physiology, 194, 406.

Bennet, M. A., and Pentecost, B. L. (1970). The pattern of onset and spontaneous cessation of atrial fibrillation in man. Circulation, 41, 981.

Goel, B. G., and Han, J. (1970). Atrial ectopic activity associated with sinus bradycardia. Circulation, 42, 853.

Han, J., Millet, D., Chizzonitti, B., and Moe, G. K. (I966). Temporal dispersion of recovery of excitability in atrium and ventricle as a function of heart rate. American Heart fournal, 71, 481.

Hecht, H. H. (1970). Mechanisms, causes, and treatment of paroxysmal atrial fibrillation. In Symposium on Cardiac Arrhythmias, p. 747. Ed. by E. Sandøe, E. Flensted-Jensen, and K. H. Olesen. AB Astra, Södertälje, Sweden.

Hecht, H. H., Katz, L. N., Pick, A., Prinzmetal, M., and Rosenblueth, A. (1953). The nature of auricular fibrillation and flutter. A symposium. Circulation, 7, 591.

Killip, T., and Gault, J. H. (1965). Mode of onset of atrial fibrillation in man. American Heart fournal, 70, 172 .

Sano, T., and Scher, A. M. (1964). Multiple recording during electrically induced atrial fibrillation. Circulation Research, 14, 117.

Scherf, D. (1966). The mechanism of flutter and fibrillation. American Heart Fournal, 71, 273.

Scherf, D., Cohen, J., and Rafailzadeh, M. (I966), Excitatory effects of carotid sinus pressure. Enhancement of ectopic impulse formation and of impulse conduction. American fournal of Cardiology, 17, 240.

Requests for reprints to Dr. Nabil El-Sherif, Cardiology Department, Faculty of Medicine, Cairo University, Cairo, Egypt, U.A.R. 